\title{
Left paraduodenal hernia: \\ Report of a case, with \\ radiographic findings, \\ including abdominal \\ computed tomography
}

\author{
STEWART D. COLE, D.O. \\ Fort Worth, Texas
}

\begin{abstract}
Paraduodenal hernia is an uncommon cause of bowel obstruction, accounting for less than 1 percent of all cases of bowel obstruction. A paraduodenal hernia should be suspected in a patient with chronic, atypical abdominal pain associated with small bowel obstruction. A case of a left paraduodenal hernia is described, with emphasis on the radiographic findings. The characteristics of the hernia on computed tomography of the abdomen are discussed. The appearance of this hernia is described as it relates to barium contrast study of the upper gastrointestinal tract.
\end{abstract}

As of January 1981, 777 cases of paraduodenal hernia had been reported in the literature. ${ }^{1}$ There is a 3:1 male-to-female predominance in the incidence of paraduodenal hernias, ${ }^{1}$ and left-sided hernias are three times more common than those on the right side. ${ }^{2}$ Paraduodenal hernia accounts for less than 1 percent of all cases of bowel obstruction. ${ }^{1}$ This type of internal hernia is associated with an anomaly of rotation and reduction of the midgut loop within the abdominal cavity, which creates a potential hernia fossa.

The possibility of paraduodenal hernia should be considered in the patient who has a chronic history of abdominal pain that does not fit a standard group of symptoms. A history suggestive of chronic smallbowel obstruction, especially when the patient's pain is exacerbated by eating, exertion, or standing erect, is suspicious for paraduodenal hernia. ${ }^{3}$

In this article, a case of left paraduodenal hernia is described, with emphasis placed on the radiographic findings. Barium contrast studies of the upper gastrointestinal tract and small bowel are the simplest, most cost-effective methods of identifying paraduodenal hernia. The characteristics of the hernia on computed tomography (CT) of the abdomen also are discussed. A review of the literature revealed only one other report ${ }^{4}$ of the CT findings of left paraduodenal hernia.

\section{Report of case}

A 33-year-old man was admitted to Fort Worth Osteopathic Medical Center on March 28,1986, with a chief complaint of recurrent, crampy, periumbilical pain. His most recent episode of abdominal pain and vomiting had begun approximately 1 hour after eating. About 4 to 5 years earlier he had been admitted to a hospital for similar abdominal problems, and a thorough diagnostic work up had failed to identify the exact cause of his pain. Evaluation by several physicians since that time also had failed to produce a definitive diagnosis. In July 1985 the patient was involved in a motor vehicle accident, from which he suffered rib fractures and pneumothorax. He denied having undergone previous abdominal surgery.

Physical examination revealed a muscular man with severe abdominal pain. His abdomen was distended. No bowel sounds were heard, and there were no signs of peritoneal irritation. The inguinal canals were free of hernia. Rectal examination revealed no mass, bleeding, or prostate abnormality.

Laboratory data at the time of admission showed a normal complete blood count and serum electrolyte, serum calcium, blood urea nitrogen, and serum creatinine levels. Other routine blood chemistry values also were normal. Urine culture revealed $>100,000 / \mathrm{cl}$. $\mathrm{ml}$. of Enterobacter organisms, which was consistent with a urinary tract infection.

A number of radiographic studies were performed (Figs. 1-6). Initial plain films of the abdomen revealed a suspicious soft-tissue mass in the mid-upper abdomen. Intravenous urography demonstrated blunted calices on the left, which suggested chronic pyelonephritis. An extrarenal pelvis also was noted on the left. The plain films' lesion was not associated with the urinary tract; instead, it was thought to be a pseudotumor of fluid-filled loops of small bowel. Abdominal CT showed dilated loops of small bowel filled with fluid and oral contrast material. These loops were confined to one area of the abdomen and appeared to be enclosed within a sac. An internal hernia was considered to be the possible etiology for these findings. A barium study of the small bowel was ordered for further evaluation, and these detailed films revealed loops of dilated small bowel confined to the left side of the 


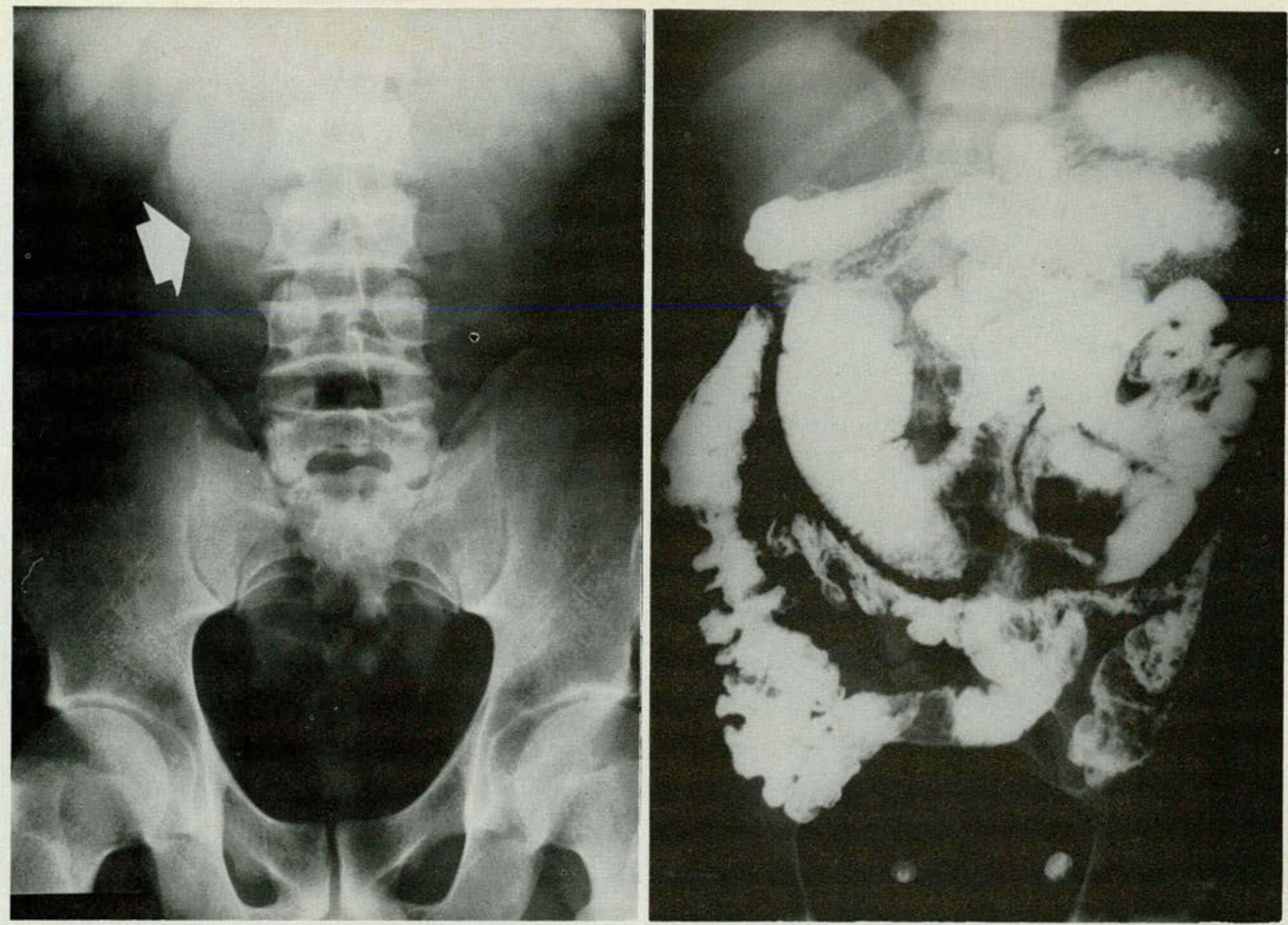

Fig. 1. Plain film of the abdomen shows the pseudotumor appearance of fluid-filled loops of small bowel contained by a hernia sac (arrow). Fig. 2. Barium study film demonstrates left paraduodenal hernia, with displaced loops of small bowel confined within a hernia sac.

abdomen. A diagnosis of left paraduodenal hernia was made.

At surgery, a defect in the left paraduodenal fossa was seen, and multiple segments of small intestine were found within it. One inflamed segment of small bowel was identified at the origin of the herniated bowel. The entire small bowel was reduced from the hernial sac, and the sac was closed at the level of the ligament of Treitz. The patient was discharged on the eighth postoperative day in good condition.

\section{Discussion}

\section{Anatomy}

Paraduodenal hernias result from a failure of the mesentery to fuse with the parietal peritoneum at the ligament of Treitz. The hernia is classified as being either left or right, depending upon its relationship to the duodenum as it emerges from its retroperitoneal position, and upon the direction in which the opening of the hernia fossa faces. ${ }^{2}$ These hernias probably originate from abnormalities of rotation and reduction of the midgut loop within the abdominal cavity; however, the exact mechanism for this remains controversial. ${ }^{1}$
A left-sided paraduodenal hernia can be located in the fossa to the left of the ascending limb of the duodenum. It can also occur in the mesocolic fossa on the left side of the abdomen. On the right side, small bowel may herniate into the posterior duodenal fossa, the mesentericoparietal fossa, or the parajejunal fossa. ${ }^{5}$

\section{Radiographic findings}

Plain films of the abdomen of a patient with acute distress may demonstrate dilated loops of small bowel confined to one area. In the present case, the fluid-filled loops of small bowel were confined to one area by the hernial sac, forming a smooth round border and having a pseudotumor appearance (Fig. 1). Pseudotumor also is associated with other causes of small-bowel obstruction and is not pathognomonic of a paraduodenal hernia.

Upper gastrointestinal and detailed small-bowel films can accurately diagnose paraduodenal hernias and also can determine whether the hernia is left or right sided. Displaced bunched loops of small bowel outlined by barium and confined within a sac 


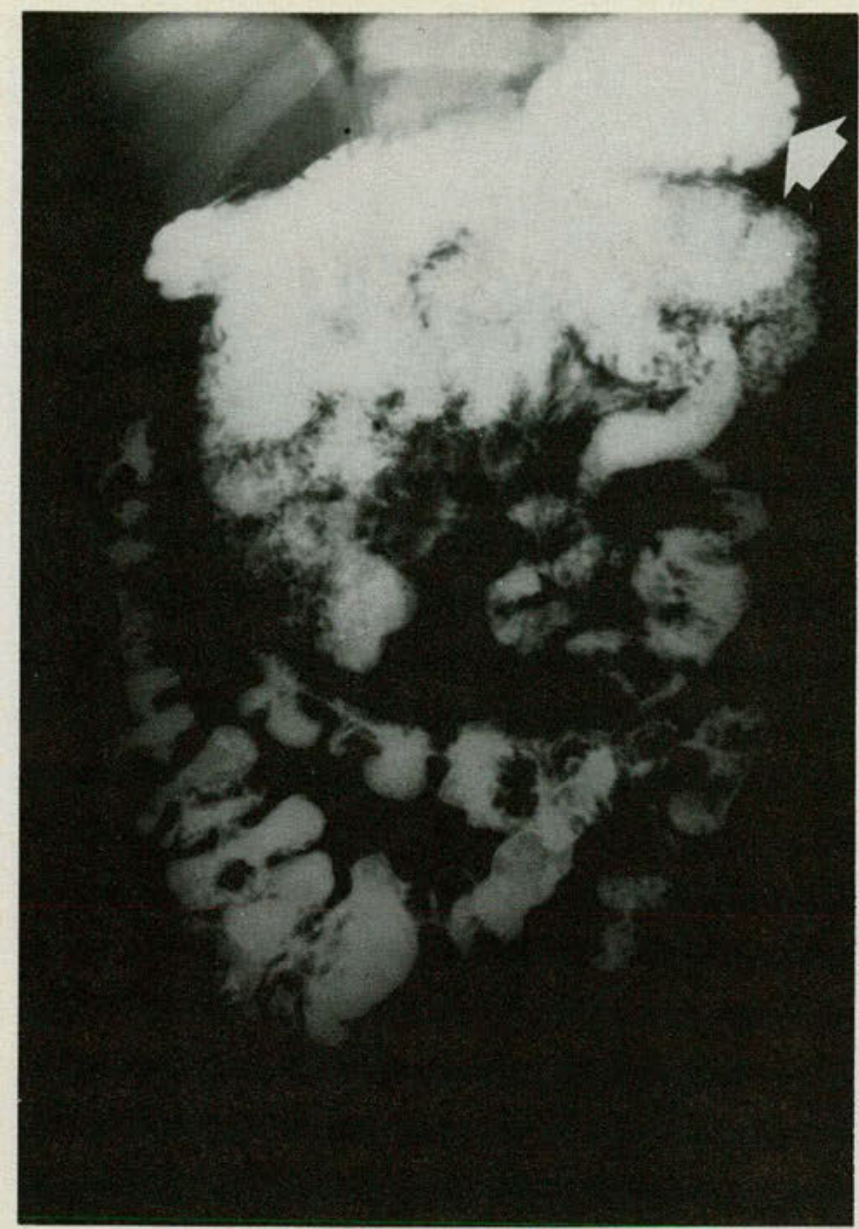

Fig. 3. Above. Detailed barium study film shows a left paraduodenal hernia displacing the stomach upward (arrow) and the transverse colon inferiorly.

Fig. 4. Upper right. CT scan of the abdomen demonstrates a contrast-filled loop of small bowel adjacent to the left psoas muscle. This finding in a patient with left paraduodenal hernia also was described by Passas and associates. 4

Fig. 5. Loops of small bowel filled with fluid and contrast medium are confined to one area of the abdomen on this CT scan. A sheet of peritoneum forms part of the hernial sac (arrow).

Fig. 6. Abdominal CT image shows thickened bands of smallbowel mesentery radiating to contrast-filled small intestine.

are seen in Figure 2. There may be delayed transit of barium in partial obstruction. A left-sided hernia may show the stomach displaced upward and to the right. The colon may be displaced to the left or inferiorly (Fig. 3). A right-sided hernia usually displaces the stomach to the left. Malrotation of the colon can be associated with a right-sided lesion, with the ascending colon displaced to the left. ${ }^{2}$

The abdominal CT features of paraduodenal hernia have seldom been described. A literature review revealed only one description of left paraduodenal hernia, that of Passas and associates, ${ }^{4}$ who docu-
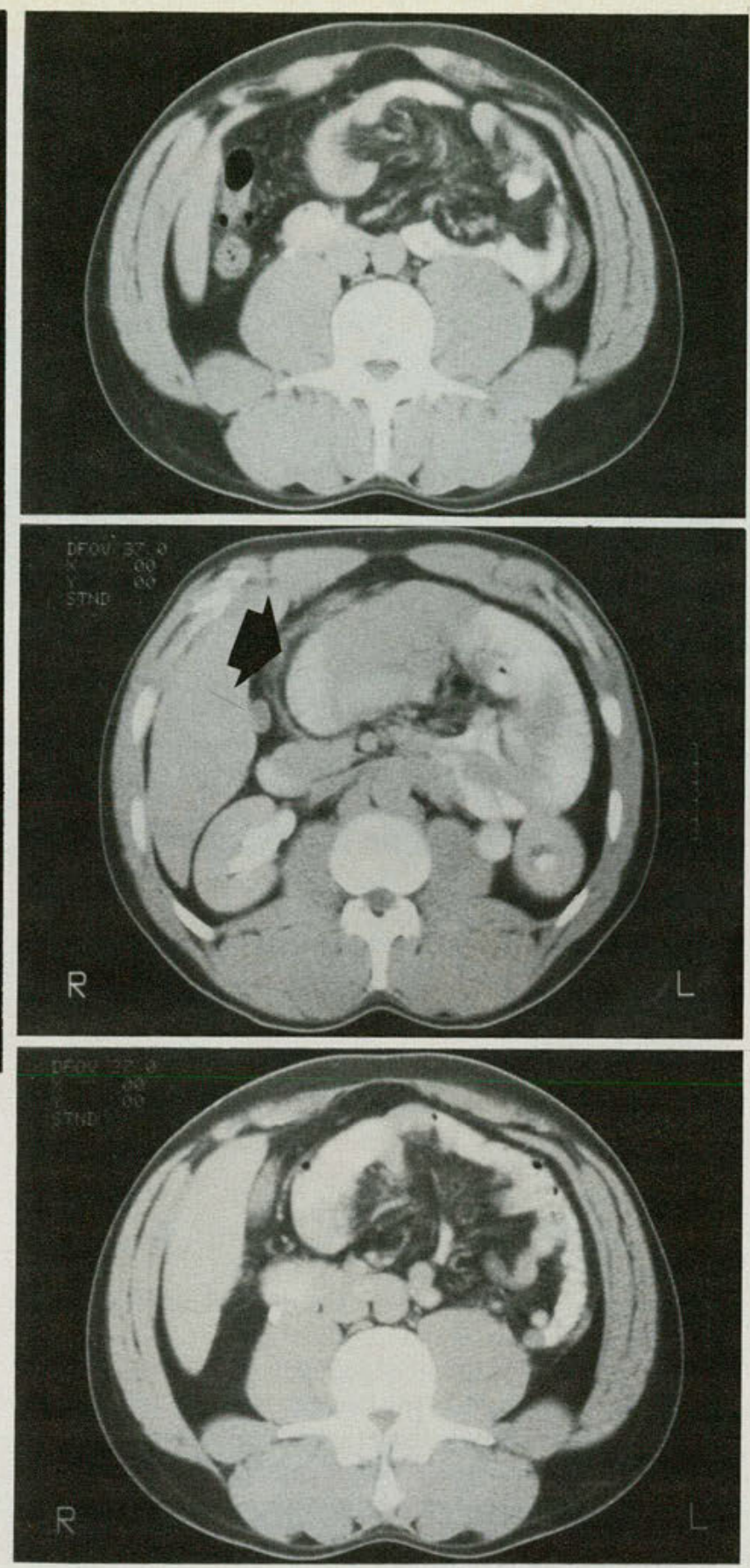

mented loops of Gastrografin-filled small bowel behind the pancreas in touch with the psoas muscle. Our computed tomography unit was able to identify loops of contrast-filled small bowel in contact with the left psoas muscle (Fig. 4). We were not able to definitely identify small bowel posterior to the pancreas, however. Other CT images showed fluid and contrast-filled loops of small bowel confined to one area of the abdomen. A thickened sheet of peritoneum forming the hernial sac was identified (Fig. 5). Other CT scans showed small bowel confined within the sac, with a thickened, centrally 
located mesentery radiating to contrast-filled small-bowel loops (Fig. 6).

\section{Summary}

Paraduodenal hernias are the cause of less than 1 percent of all bowel obstructions. They should be considered in cases of chronic abdominal pain of undetermined etiology.

The basic studies for diagnosis are the barium contrast upper gastrointestinal and detailed smallbowel examinations. Computed tomography descriptions of paraduodenal hernia are uncommon. In the case reported here, CT images displayed loops of small bowel in contact with the left psoas muscle. Contrast-filled small bowel confined within a sac of peritoneum and thickened mesentery that radiated to the small bowel were identified.
Appreciation is expressed to E. Wayne Johnson, D.O., for his help in evaluating the CT images included in the paper.

1. Berardi, R.S.: Paraduodenal hernias. Surg Gynecol Obstet 152:99-110, Jan 81

2. Marshak, R.H., and Lindner, A.E.: Radiology of the small intestine. W.B. Saunders Co., Philadelphia, 1970, pp. 473-7

3. Alexander, F.K.: Roentgen diagnosis of intra-abdominal hernia. Am Roentgenol 38:92-101, Jul 37

4. Passas, V., et al.: Computed tomography of left paraduodenal hernia. $J$ Comput Assist Tomogr 10:542-3, May-June 86

5. Estrada, R.L.: The paraduodenal hernias. Can J Surg 25:7-8, Jan 82

Dr. Cole is a radiology resident, Fort Worth Osteopthic Medical Center, Fort Worth, Texas. S.R. Briney, D.O., is chief, Department of Medical Imaging, and director, Radiology Resident Training, FWOMC.

Dr. Cole, Fort Worth Osteopathic Medical Center, 1000 Montgomery Street, Fort Worth, Texas 76107. 